\title{
Juxta-Articular Myxoma
}

National Cancer Institute

\section{Source}

National Cancer Institute. Juxta-Articular Myxoma. NCI Thesaurus. Code C6580.

A rare myxoma that usually arises in an area close to a large joint. Morphologically it resembles a cellular myxoma. In contrast to an intramuscular myxoma, it is not associated with mutations of the GNAS gene. 